\title{
Role of Magnetic Fields in Ram Pressure Stripped Galaxies
}

\author{
Ancla Müller 1,*(D), Alessandro Ignesti ${ }^{2} \mathbb{D}$, Bianca Poggianti ${ }^{2} \mathbb{D}$, Alessia Moretti ${ }^{2} \mathbb{D}$ and Mpati Ramatsoku ${ }^{3,4} \mathbb{D}^{\mathbb{D}}$ \\ and Ralf-Jürgen Dettmar ${ }^{1}$ (D)
}

1 Faculty of Physics and Astronomy, Astronomical Institute, Ruhr University Bochum, Universitätsstr. 150, 44801 Bochum, Germany; dettmar@astro.rub.de

2 INAF-Osservatorio Astronomico di Padova, Vicolo dell'Osservatorio 5, I35122 Padova, Italy; alessandro.ignesti@inaf.it (A.I.); bianca.poggianti@oapd.inaf.it (B.P.); alessia.moretti@inaf.it (A.M.)

3 Department of Physics \& Electronics, Rhodes University, Makhanda 6139, South Africa; mpati.ramatsoku@inaf.it

4 INAF-Osservatorio Astronomica di Cagliari, Via della Scienza 5, I09047 Selargius, Italy

* Correspondence: amueller@astro.rub.de

Citation: Müller, A.; Ignest, A.; Poggianti, B.; Moretti, A.; Ramatsoku, M.; Dettmar, R.-J. Role of Magnetic Fields in Ram Pressure Stripped Galaxies. Galaxies 2021, 9, 116. https://doi.org/10.3390/ galaxies 9040116

Academic Editor: Dimitris M. Christodoulou

Received: 17 November 2021 Accepted: 6 December 2021 Published: 11 December 2021

Publisher's Note: MDPI stays neutral with regard to jurisdictional claims in published maps and institutional affiliations.

\section{Copyright: (c) 2021 by the authors.} Licensee MDPI, Basel, Switzerland. This article is an open access article distributed under the terms and conditions of the Creative Commons Attribution (CC BY) license (https:/ / creativecommons.org/licenses/by/ $4.0 /)$.

\begin{abstract}
Ram-pressure stripping is a crucial evolutionary driver for cluster galaxies and jellyfish galaxies characterized by very extended tails of stripped gas, and they are the most striking examples of it in action. Recently, those extended tails are found to show ongoing star formation, raising the question of how the stripped, cold gas can survive long enough to form new stars outside the stellar disk. In this study, we summarize the most recent results achieved within the GASP collaboration to provide a holistic explanation for this phenomenon. We focus on two textbook examples of jellyfish galaxies, JO206 and JW100, for which, via multi-wavelength observations from radio to X-ray and numerical simulations, we have explored the different gas phases (neutral, molecular, diffuse-ionized, and hot). Based on additional multi-phase gas studies, we now propose a scenario of stripped tail evolution including all phases that are driven by a magnetic draping sheath, where the intracluster turbulent magnetized plasma condenses onto the galaxy disk and tail and produces a magnetized interface that protects the stripped galaxy tail gas from evaporation. In such a scenario, the accreted environmental plasma can cool down and eventually join the tail gas, hence providing additional gas to form stars. The implications of our findings can shed light on the more general scenario of draping, condensation, and cooling of hot gas surrounding cold clouds that is fundamental in many astrophysical phenomena.
\end{abstract}

Keywords: galaxies: magnetic fields; galaxies: evolution; galaxies: ISM; radio continuum: galaxies; polarization

\section{Introduction}

On cosmic scales, star formation activity in galaxies has strongly declined since $\mathrm{z} \sim 2$ such that a large number of galaxies have evolved into passive galaxies while the star formation rate at fixed stellar mass has decreased [1]. In order to understand the drop in star formation rate density of the Universe [2], it is necessary to study the processes of gas acquisition and loss, which can be divided into internal and external mechanisms. Gas consumption as well as stellar or Active Galactic Nuclei (AGN) feedback processes feeding the galaxy halo can result in a decrease in star formation rate. There are several external mechanisms depleting the gas content of a galaxy [3], and their efficiency depends on whether the galaxy is isolated, is part of a group, or part of a cluster. One of the most efficient environmental mechanisms is ram pressure stripping, which has been observed via neutral hydrogen studies in galaxy clusters [4-8]. A varied situation is found in galaxy groups [9-11].

More recently, extreme ram pressure stripped galaxies with long gaseous tails, named jellyfish galaxies, were found moving at high speed in a variety of galaxy clusters, e.g., [12,13]. Significant ongoing star formation has been detected within galactic disks and far out 
into the tails $[14,15]$. This demonstrates that the process of gas removal is not always associated with the immediate quenching of star formation. On the contrary, the exact interplay between gas stripping and star formation activity might vary from galaxy to galaxy depending on their size, location and orbit within a cluster, as well as on cluster properties. Understanding this interplay is crucial for fully understanding how star formation is eventually quenched in dense environments.

Among the most compelling open questions includes the origin of the in situ star formation in ram pressure stripped tails and whether magnetic fields promote star-formation activity in the tails. Magnetic fields can play a key role in star formation processes: On parsec scales, molecular clouds collapse through magnetic field transport of angular momentum [16]. Spiral galaxies show large-scale magnetic fields along and between optical spiral arms and in their halos [17,18], anchoring the magnetic field to molecular clouds [19]. Until recently, the magnetic field of ram pressure stripped galaxies was only observed in the close vicinity of galaxy discs in nearby galaxy clusters, e.g., [20-22]. By measuring radio continuum brightness and spectral index trend in direction of the galaxy tail, these works found an excess in radio emission that cannot be explained by the current star formation in the $\mathrm{H} \alpha$ emitting knots. This can be the result of the ram-pressure stripping process in agreement with the steepening of the spectral index with distance to the galaxy disks, which is expected in a scenario where electrons travel from the disk to the tail and release their energy, indicating standard electron cooling. Based on such standard cooling assumptions, Ref. [20] predicted a magnetic field strength of about $5 \mu \mathrm{G}$ in the galaxy tails by assuming several parameters. This value is found to agree with more recent extreme ram pressure stripped tails [23,24]. Müller et al. [23] studied for the first time the magnetic field strength and configuration of both the disk and the tail of an extreme ram pressure stripped galaxy, JO206.

Long co-spatial radio continuum and $\mathrm{H} \alpha$ tails in several more ram pressure stripped galaxies have been found $[20,22,24,25]$ (Müller et al., in prep), suggesting that such tails are common. The number of known spiral galaxies with radio continuum tails, i.e., evidence of a magnetic field extended beyond the stellar disk for tens of kpc, has greatly increased recently thanks to the progress of the LOFAR Two-metre Sky Survey [26] (LoTSS). Based on the LoTSS observations, $[27,28]$ reported the discovery of hundreds of radio jellyfish in low$z$ clusters and groups. These results indicate that the formation of radio tails is ubiquitous in clusters and showed that LOFAR can be a powerful instrument for identifying ram pressure stripped galaxies across extremely wide fields.

\section{The GASP Survey}

The GAs Stripping Phenomena in galaxies $\left(\mathrm{GASP}^{1}\right)$ survey [29] has assembled a multiwavelength dataset of jellyfish galaxies in low-z clusters. Within a Multi Unit Spectroscopic Explorer (MUSE) European Southern Observatory (ESO) large program, GASP has obtained integral-field optical spectroscopy of 114 galaxies (ram-pressure stripped galaxy candidates and control sample) at $z=0.04-0.07$ with masses from $10^{9} \mathrm{M}_{\odot}$ to $10^{11.5} \mathrm{M}_{\odot}$ in clusters, groups, filaments, and isolated. These sources were selected based on unilateral debris or tails in B-band images (and no signs of it for the control sample) detected in the WINGS and OmegaWINGS surveys [30].

In order to study cold neutral gas in the galaxy disk and tails GASP also assembled HI observations from the Karl G. Jansky Very Large Array (VLA), MeerKAT, and Australia Telescope Compact Array (ATCA) of a sub-sample of GASP galaxies [31-33] (Tirna et al., submitted). We further observed the radio continuum using the same instruments and also the LOw Frequency ARray (LOFAR) [23,25] (Ignesti et al. submitted, Müller et al., in prep). In order to study the magnetic field structure, we performed deep polarization observations with the VLA and ATCA for five ram-pressure stripped galaxies; however, only one observation is already completed [23] (JO206 see below). GASP also collected Atacama Pathfinder EXperiment (APEX) CO(2-1) [34], Atacama Large Millimeter/submillimeter Array (ALMA) $\mathrm{CO}(2-1)$ and $\mathrm{CO}(1-0)$ [35] data to study molecular gas components in 
disks and tails, Ultra Violet (UV) data with Ultra-Violet Imaging Telescope (UVIT) on the AstroSat, and UV to I-band data with the Hubble Space Telescope (HST). Finally, GASP has been awarded a deep Chandra X-ray Observatory (CXO) observation of JO206 and utilised archival CXO data to study the cluster environment [23] and X-ray emission of extreme ram-pressure stripped galaxies $[25,36] .^{2}$

By assembling the different gas phases and magnetic field properties of a sub-sample of GASP galaxies, we aim to study galaxy evolution by addressing the following questions: What is the baryonic cycle between the different gas phases and the star formation in the disk and tails of ram pressure stripped galaxies? Where, how, and why is gas removed from galaxies and what is the effect on galaxy star formation? Is there a possible connection between ram pressure and the appearance of AGNs in the galaxy centers? Does ram pressure cause the quenching of star-formation on long term scales and the transformation to passive early type galaxies? What is the impact of ram pressure in groups and filaments? In this study, we focus on the recent results from the GASP collaboration about the properties of the different gas phases and how they can survive long enough in tails to evolve into stars focused on recent multi-phase gas, radio continuum, and linear polarization results collected within GASP.

\section{Multi-Phase Gas}

Jellyfish galaxies are found to be Hi deficient [31-33] (Deb et al., in prep) but contain star-forming clumps with reservoirs of $\mathrm{H}_{2} \sim 4$-times higher than expected for normal starforming galaxies of similar mass $[34,35]$. In addition, the amount of neutral + molecular gas is found to be comparable to normal star-forming galaxies of similar galaxy mass indicating (1) an effective conversion from $\mathrm{HI}$ to $\mathrm{H}_{2}$ [35] and (2) either no significant evaporation of the cold gas into the ICM or an effective accretion and mixing of hot ICM that can cool providing an additional reservoir of gas or (probably) a combination of both supported by the magnetic field configuration in the galaxy tails (see below).

The diffuse ionized gas has been studied for 71 GASP galaxies: both ram-pressure stripping candidates and a control sample [37]. We find that in the stripped tails (and even more significantly in the most extended tails such as JO206 and JW100), the OI/H $\alpha$ ratio reaches values that cannot be explained by the current star-formation alone. It is likely that at least a part of the ionization is caused either by shocks (no direct evidence found in the X-ray observations yet) or accretion of hot ICM gas and cooling and/or mixing.

Studies of GASP galaxies [25,36], as well as other objects, e.g., [38], revealed that jellyfish galaxies can host a hot phase $\left(T \simeq 10^{6}-10^{7} \mathrm{~K}\right)$ with a metallicity tentatively consistent with $\mathrm{ICM}$ values $\left(Z \simeq 0.3-0.4 Z_{\odot}\right)$ that emits $X$-rays via thermal bremsstrahlung and closely follows the morphology of the stripped $\mathrm{H} \alpha$-emitting tail. These results are in agreement with those reported for other jellyfish galaxies (see [38] and references therein). The high temperature of this phase and the generally low metallicity might suggest that this X-ray emitting phase is mostly composed by cooled ICM, thus supporting the idea that jellyfish galaxies are actually able to accrete hot, magnetized ICM during their orbits of the cluster. However, the nature of the interplay between ISM and ICM that ultimately results in the formation of the $\mathrm{X}$-ray extended emission is still unclear.

On a more general view, the most remarkable jellyfish galaxies that experience in situ star formation in extended gas tails are found to be the most massive disk galaxies in the clusters and move at very high velocities with respect to the intracluster medium [39]. The high total mass, therefore, allows the galaxy to hold onto its gas until it has reached the densest part of the ICM, which is also an important factor to understand the lifetime and gas phases in the extended ram-pressure stripped tails. The high ICM pressure can effectively build a magnetic sheath protecting the gas from evaporation into the ICM at the tail edges (observed in case of JO206, see below). In the following, we summarize the most relevant results achieved for two of the most studied galaxies of the gasp survey, JO206 and JW100. 
JO206 is a textbook case galaxy for studying ram-pressure stripping due to its $90 \mathrm{kpc}-$ long $\mathrm{H} \alpha$-emitting ionized gas tail [29]. JO206 is part of the low-mass cluster IIZW108 $(\sigma=575 \mathrm{~km} / \mathrm{s})$ at a redshift of $z=0.05133$, and it has a total stellar mass of $M_{\star}=$ $7.8 \times 10^{10} \mathrm{M}_{\odot}$ and a star-formation rate of $4.8 \mathrm{M}_{\odot} /$ year and $0.5 \mathrm{M}_{\odot} /$ year in its disk and tail, respectively. It is falling into the cluster with a velocity of at least $1200 \mathrm{~km} / \mathrm{s}$ at a projected distance of $300 \mathrm{kpc}$ from the cluster center. The multiple star-forming clumps in the galaxy disk and tail were identified based on their $\mathrm{H} \alpha$ emission. These clumps are generally found to coincide also with a reservoir of molecular gas [34,35] (left panel in Figure 1) and neutral hydrogen [31] (right panel in Figure 1). The molecular gas follows the clumpy structure of the star-forming knots. The lower spatial resolution Hi observations show the presence of $\mathrm{HI}$ along the entire $\mathrm{H} \alpha$-emitting tail. However, the total amount of neutral + molecular gas was found to be normal [35]. In Tomicic et al. [37], we found an additional ionized gas component that cannot be associated with the current star formation in the galaxy disk and tail.
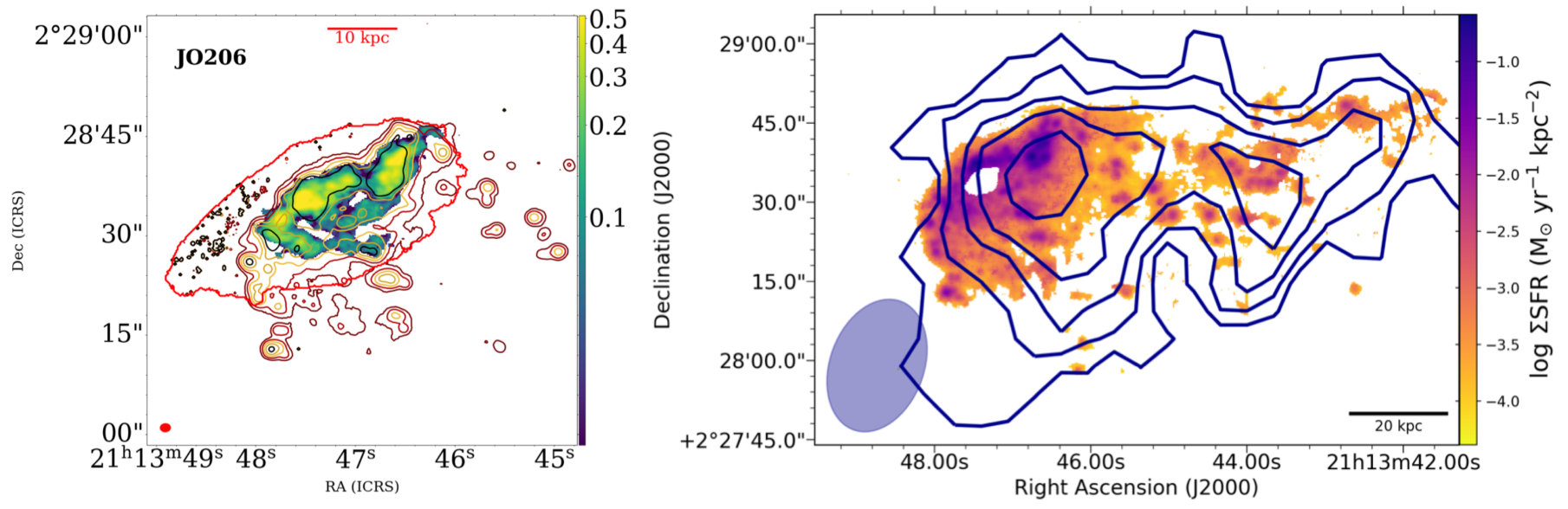

Figure 1. Multi-phase gas components of JO206. Left: Molecular gas CO(1-0) image in units Jy/beam.km/s and $\mathrm{H} \alpha$ ionised gas contours taken from Moretti et al. [35]. The stellar disk is shown as a red contour. Reproduced with permission from A. Moretti, published by AAS (ApJ), 2020. Right: Star formation rate surface density image and HI neutral gas contours taken from Ramatsoku et al. [31]. The Hi beam is shown in the left corner.

The jellyfish galaxy JW100, at a redshift of 0.06189 , is part of the A2626 cluster $(\sigma=650 \mathrm{~km} / \mathrm{s})$ [15]. It is located at a projected distance of $100 \mathrm{kpc}$ from the cluster center and is falling into the cluster with a line-of-sight velocity of $1800 \mathrm{~km} / \mathrm{s}$. The galaxy has a stellar mass of $M_{\star}=2.9 \times 10^{11} \mathrm{M}_{\odot}$, which makes it the most massive galaxy in the GASP sample, and forms effectively stars in its disk and tail, mostly in the southern tail region, with a star-formation rate of $3.2 \mathrm{M}_{\odot} /$ year and $0.8 \mathrm{M}_{\odot} /$ year, respectively. Multiple gas phases of JW100 are already coherently discussed in Poggianti et al. [25] for the galaxy disk and the $50 \mathrm{kpc}$-long $\mathrm{H} \alpha$-emitting gas tail. The star-forming regions coincide with $\mathrm{H} \alpha$, UV, and CO clumps (left panel in Figure 2). However, not all observables are detected in all clumps at the same time, and this can be interpreted as a star-formation sequence. The amount of molecular gas is also found to be quite high [35] and the diffuse ionized gas cannot be explained by star formation alone [37]. Finally, Chandra X-ray observations revealed that JW100 has an extended X-ray emission elongated along the tail (right panel in Figure 2). The temperature of this component is $\sim 1 \mathrm{keV}$, which is not consistent with either the temperature of the surrounding ICM [40] $(k T=3.5 \mathrm{keV})$ or the typical hot ISM phase $(k T \simeq 0.1-0.2 \mathrm{keV}$, e.g., [41]). Interestingly, X-ray luminosity is higher than expected based on the current SFR, thus suggesting that the hot gas enveloping JW100 was not only heated up by star formation. 

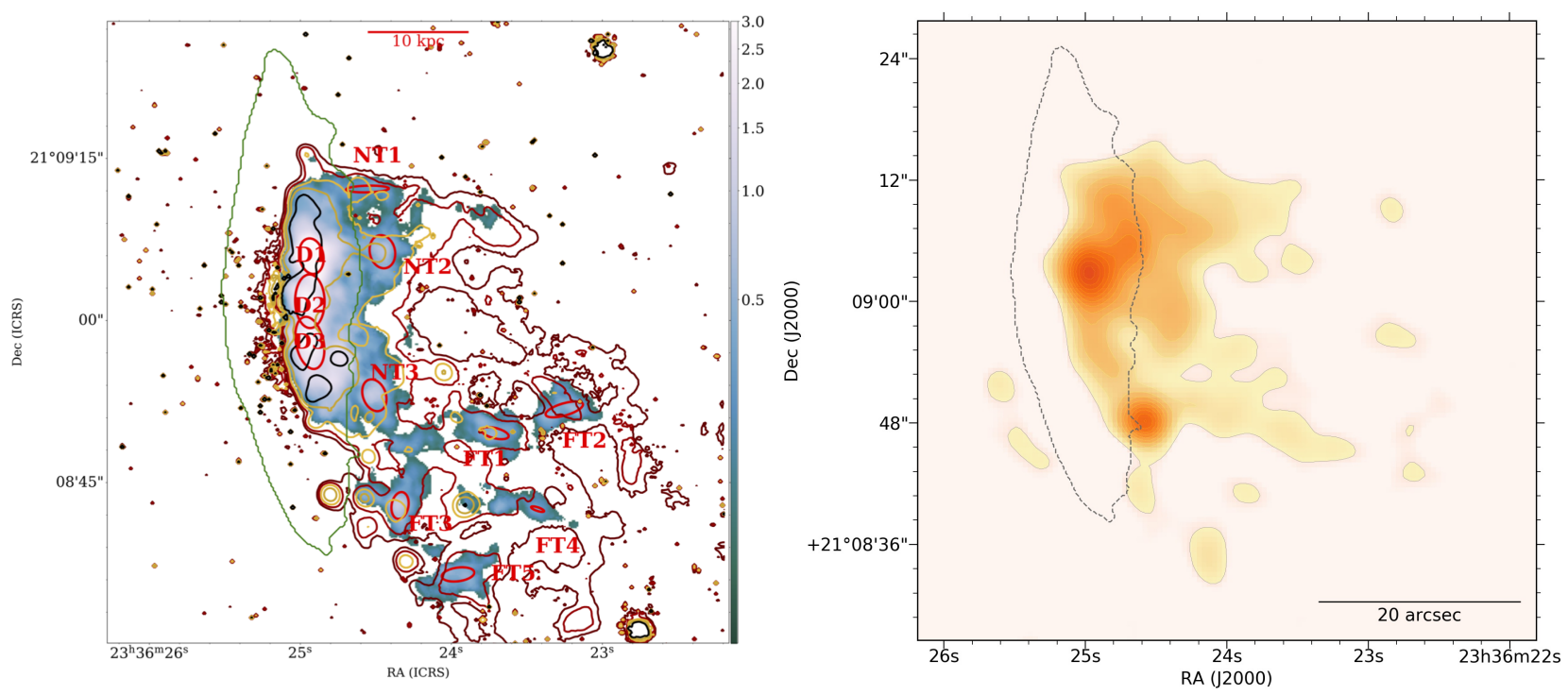

Figure 2. Multi-phase gas component of JW100: Left: Molecular gas CO(2-1) image in units Jy/beam.km/s and H $\alpha$ ionized gas contours taken from Moretti et al. [42]. The stellar disk is shown in green contours. Reproduced with permission from A. Moretti, published by AAS (ApJ), 2019. Right: Hot gas (X-ray) in orange and stellar disk is shown in black contours.

\section{Radio Continuum and Polarization Observations}

Ram pressure stripped galaxies have been observed in radio continuum over the past decades. Within GASP, we conducted two case studies to grasp the role of radio continuum and the corresponding magnetic field strength not only in the host galaxy disks but also in the extended jellyfish tails of JO206 [23] and JW100 [24,25]. In the first mentioned study, we were additionally able to study for the first time the magnetic field configuration far into the extended galaxy tail. Here, we provide a summary of both case studies showing only the most important observational findings. The theoretical implications we drew from them are given in the next section.

In Müller et al. [23], deep VLA C-arrays $2.7 \mathrm{GHz}$ in full polarization mode were combined with VLA C-array $1.4 \mathrm{GHz}$ observation [31] to study the spectral index and magnetic field properties in the $90-\mathrm{kpc} \mathrm{H} \alpha$ emitting, ionized gas tail of JO206 selected from GASP. In Figure 3, we show that the extent of the tail in radio continuum and polarization exceeds $40 \mathrm{kpc}$ at $2.7 \mathrm{GHz}$, which is one of the most extreme ram-pressure stripped galaxy tail forming in situ stars in the radio range reported so far.
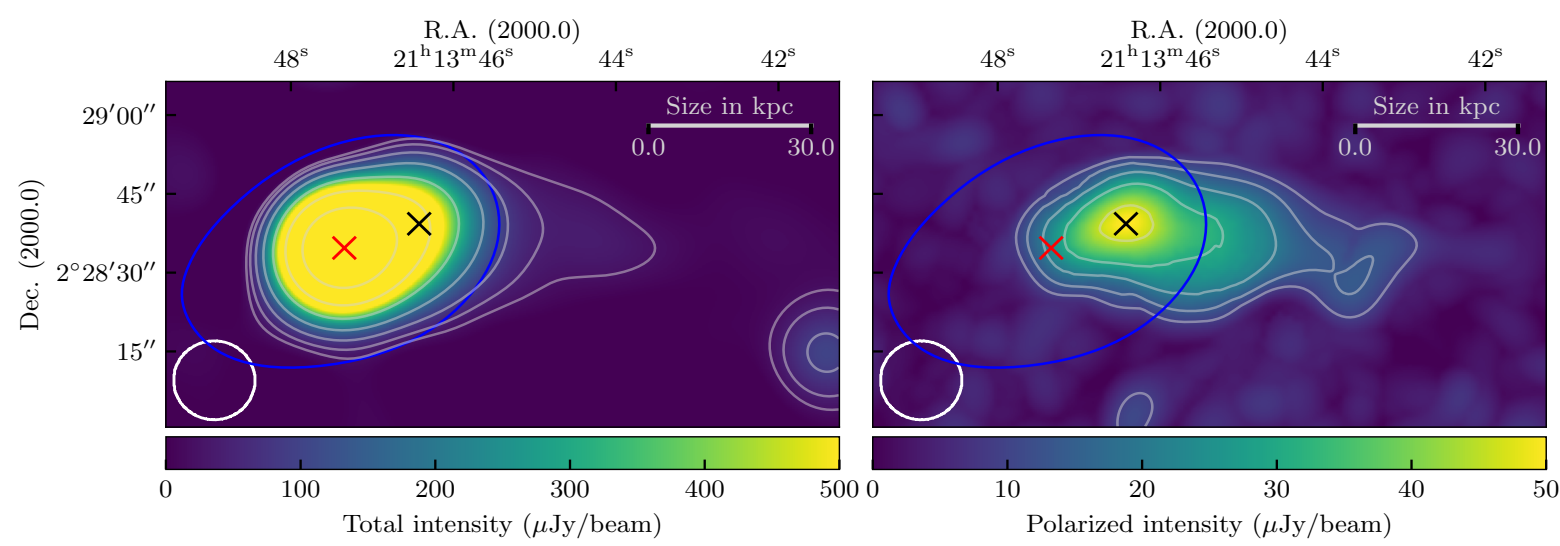

Figure 3. Radio emission of JO206 at 2.7 GHz adapted from Müller et al. [23]. Left: Radio continuum emission and radio continuum contours with its peak shown in red. The radio beam is shown in the left corner, and the stellar disk convolved to the radio resolution is shown in blue contours. Right: Linear polarized emission and linear polarized emission contours with its peak shown in black. 
We find the radio disk properties to be in general agreement with star-forming galaxies-a spectral index of $\alpha \approx-0.7$, a magnetic field strength of $B \approx 7 \mu \mathrm{G}$, and a polarized fraction of $2 \%$-which is in agreement with previous studies, while the tail appears significantly different. The tail spectral index is found to be steepening with distance from the galaxy disk, reaching a mean value of about -2 (top right panel in Figure 4), indicating a significant aging of electrons through the tail. We investigated different methods (equipartition, electron cooling length, and magnetic draping) to restrict the tail magnetic field to $B \approx(2-4) \mu \mathrm{G}$. The combination of high degree of fractional polarization of about $54 \%$ (bottom right panel in Figure 4) and the derived polarization angle (corrected for Faraday rotation) revealed an aligned large scale magnetic field along the length of the tail in the stripping direction (left panel in Figure 4).
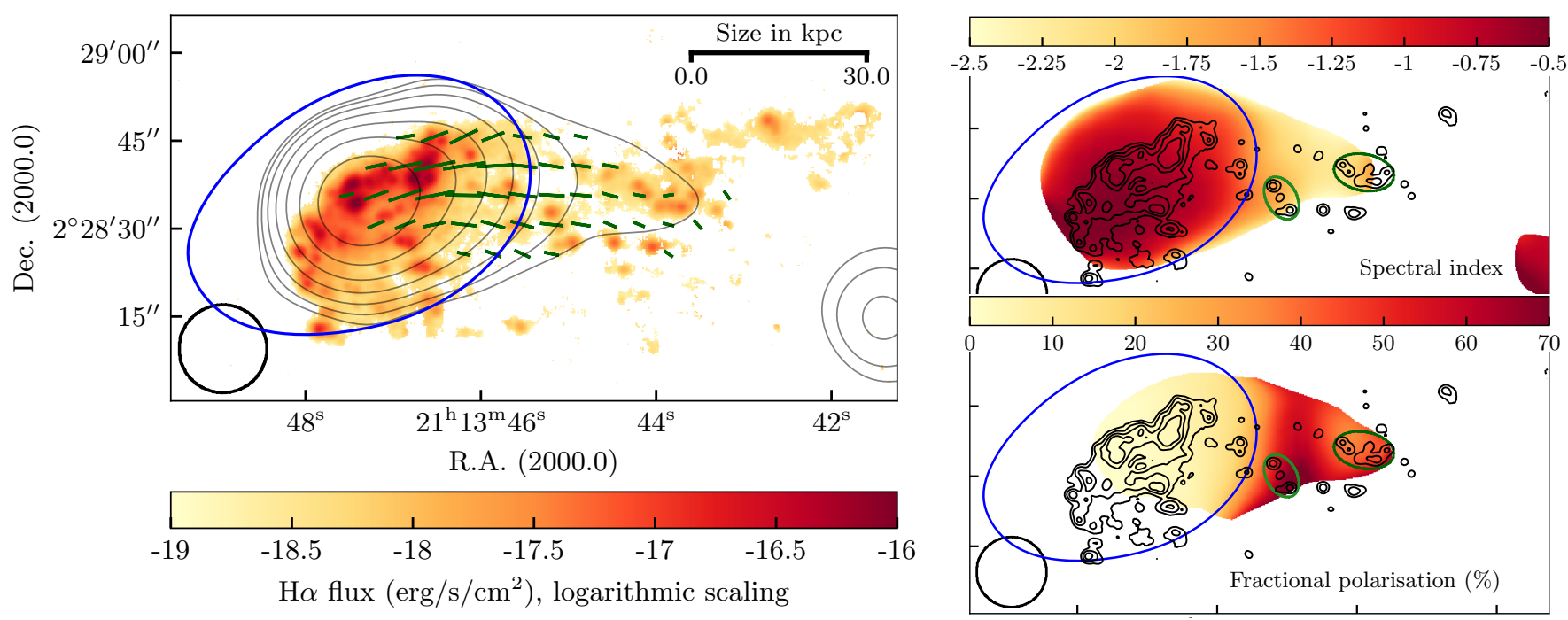

Figure 4. Radio observables of JO206 at 2.7 GHz adapted from Müller et al. [23]. Left: $\mathrm{H} \alpha$ ionised gas image and magnetic field vectors in green. The radio beam is shown in the left corner and the stellar disk convolved to the radio resolution is shown in blue contours. Top right: Spectral index image between $2.7 \mathrm{GHz}$ and $1.4 \mathrm{GHz}$ and $\mathrm{H} \alpha$ ionized gas contours. Bottom right: Fractional polarization and $\mathrm{H} \alpha$ ionized gas contours.

We identified star-forming knots in the tail (green regions in Figure 4) with spectral index flattening and reduced fractional polarizations that, however, were not able to disrupt the highly ordered magnetic field. The latter are aligned with the tail and illuminated by aged electrons with a steep spectral index. Such ordered magnetic fields may prevent heat and momentum exchange and favor in situ star formation in the tail. In this scenario, star formation in the tail should be a self-regulating process: A strong and widespread starformation activity would disrupt the ordered magnetic field, which in turn is fundamental for allowing the star-formation process to continue. The study of JO206 is unique and of general interest to understand the impact of magnetic fields during ram pressure stripping and star formation. So far, such a configuration of spectral index steepening, high fractional polarization, and aligned magnetic field was only found in head-tail galaxies (AGN driven jets experiencing ram pressure stripping) (see [43] and references therein). This recent study of JO206 puts forward the idea that such a combination can also be relevant to understand the star-forming, ram-pressure stripped tails in galaxy clusters. Müller et al. [23] also provide a comparison to magneto-hydrodynamical simulations to evaluate the origin of the radio emission. We provide this picture in the next section.

JW100 also shows an extended, non-thermal radio emission. Although a detailed polarimetric analysis has not been carried out yet, its emission has been detected in a wider range of frequencies than JO206, extending from $144 \mathrm{MHz}$ to $5.5 \mathrm{GHz}$ [44-46]. A combined analysis has recently been investigated within the GASP collaboration involving, for the first time, LOFAR, MeerKAT and VLA observations [24]. Overall, radio emission follows 
the morphology of both the $\mathrm{H} \alpha$ and X-ray emission (Figure 5). It is truncated with respect to the stellar disk and extends linearly along the stripped tail, thus supporting the idea that non-thermal radio emission (due to the presence of the magnetic field) is affected by ram-pressure stripping [24]. The synchrotron spectrum revealed that the relativistic electrons are accelerated within the disk and then displaced by ram pressure stripping, in accordance with the ram pressure stripping scenario. Thus, similarly to the case of JO206, a spectral steepening trend has also been observed for JW100. Moreover, the comparison between the continuum spectrum and the $\mathrm{H} \alpha$ emission allowed us to constrain the time scale of the star formation quenching [24].

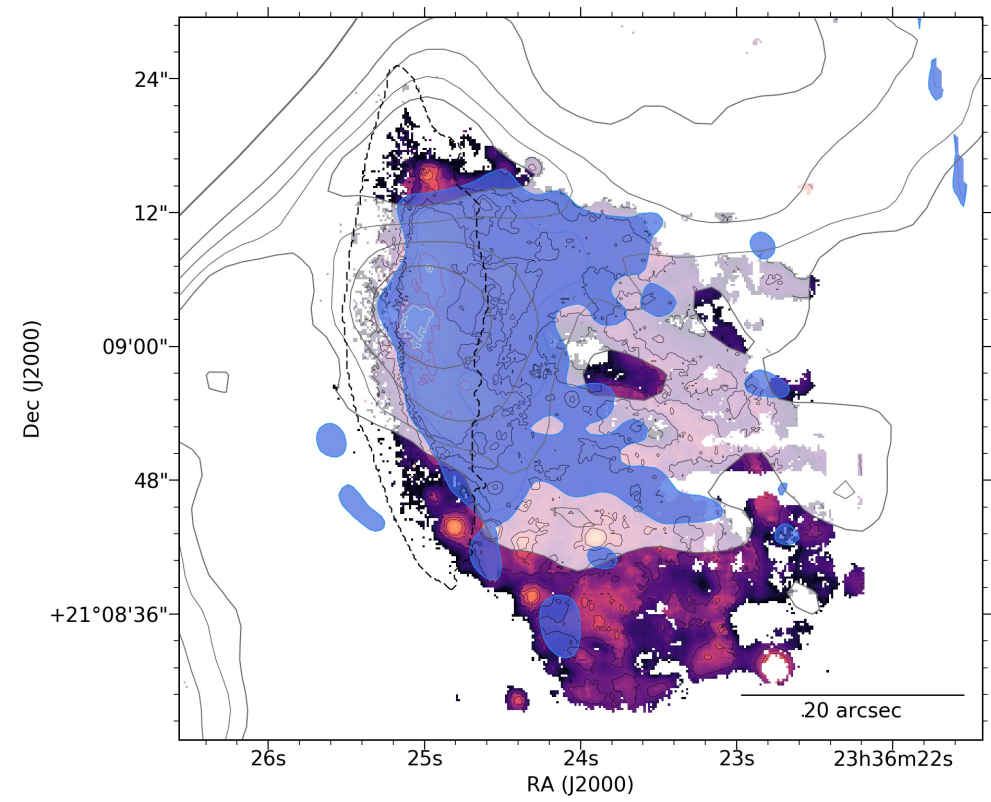

Figure 5. Composite image of MUSE $\mathrm{H} \alpha$ (red, $0.2^{\prime \prime}$ resolution), Chandra X-ray (blue contour, $1.5^{\prime \prime}$ resolution), and LOFAR radio continuum at $144 \mathrm{MHz}$ (grey contours at 3,6,12, $24 \times \mathrm{rms}$, $\mathrm{rms}=94 \mu \mathrm{Jy} /$ beam, $8.8^{\prime \prime} \times 6.4^{\prime \prime}$ resolution) of JW100 from Ignesti et al. [24]. Reproduced with permission from A. Ignesti, published by AAS (ApJ), 2021.

In agreement with the findings for JW100 and JO206, the radio continuum in rampressure stripped galaxies is found to be co-located with $\mathrm{H} \alpha$ not only within the disk but also with the extended, $\mathrm{H} \alpha$-emitting tails, namely JO204, JO194, JO175, and JO147 (Müller et al., in prep).

\section{Theoretical View}

Theoretical assumptions, simulations, and their comparison to observations of rampressure stripped galaxies have provided several approaches to explain the enhanced radio continuum in galaxy disks and tails, the appearance of the different gaseous phases, and the magnetic field strength and configuration. In the literature, there have been a few simulations of ram-pressure stripped galaxies including magnetic fields over the last decade. Tonnesen and Stone [47] found that the stripping rate of the disk gas was not significantly affected by galaxy magnetic field strength and morphology while RamosMartínez et al. [48] proposed a reduced compressibility of the magnetized plasma that makes the tail gas less clumpy and filamentary-like in comparison to non-magnetized studies. Vollmer et al. [49] found asymmetric polarized ridges that can be explained by adiabatic compression of galaxy magnetic fields at the leading edge. In addition, galaxies are expected to sweep up the intracluster magnetic field as they orbit inside a galaxy cluster and develop a magnetic draping sheath [50]. Such a sheath is lit up with cosmic rays originating from supernovae and generates coherent polarized emission at the leading edges of the galaxy [51]. Importantly, Ruszkowski et al. [52] proposed that 
simulated galaxies interacting with a magnetized ICM show highly ordered magnetic fields in the tails that suppress strong Kelvin-Helmholz instabilities at the interface between the stripped gas and the ICM [53].

In Müller et al. [23] we have performed state-of-the-art simulations with the moving mesh code AREPO [54-56] of a galaxy-sized, cold-dense spherical cloud that interacts with a hot-diffuse ICM (see also [57]) to model synchrotron characteristics. We adopt a scenario in which the electrons are accelerated by supernovae into the galaxies' disk and propagate into the surrounding interstellar medium. While the medium experiences ram-pressure stripping, the electrons are carried into the galaxies' tail and cool by synchrotron emission and inverse Compton interactions with the cosmic microwave background photons. In order to grasp the essential physics, cloud size, wind field topologies, wind velocity, and cloud-wind density were adjusted to match the observations. The supersonic wind the cloud is facing has, therefore, been divided into three scenarios: (1) a purely hydrodynamical wind, (2) a wind composed of a homogeneous magnetic field, and (3) a turbulent magnetized wind. The density of the cloud is chosen to be a factor of $10^{3}$ higher than the wind and is in pressure equilibrium with the surroundings, and we adopted a weak magnetic field by assuming a thermal-to-magnetic pressure ratio of ten. In order to emulate synchrotron characteristics, we modeled the electron transport by identifying stripped gas cells as part of the galactic gas via Lagrangian trajectories. We follow the cooling of the electrons during the stripping process with a standard cooling function (see Methods and Supplementary Information in for any more details [23]).

In the hydrodynamical case, we found Kelvin-Helmholtz instabilities growing small perturbations at the wind-cloud interface and eventually disrupted the cloud. Therefore, in the case of both magnetized winds, we found the formation of a magnetic draping sheath protecting the cloud from disruption forming magnetized filaments in the wake of the cloud (gaseous tail) [50,53]. Here, case (3) was found to provide the best protection for the ram-pressure stripped gaseous tail. While we found the gas cloud to sweep up magnetised ICM, building a draping layer around the head, a magnetised dense gaseous tail was formed via condensation of the ICM [57]. Thereby, the magnetic field is stretched and aligns with the tail (stripping direction). In addition, for large clouds (comparable to galaxy sizes), the cold phase in the ram-pressure stripped tail is found to survive and even increased due to gas accretion from the hot wind by mixing it into a layer with intermediate temperatures $\left(T \sim 3 \times 10^{5} \mathrm{~K}\right)$, from where it quickly cools and grows the cold tail [57-59].

We further simulated synchrotron observables at $2.7 \mathrm{GHz}$, comparable to the observation frequency of JO206, and found a gas cloud facing a turbulent magnetized wind to be the best fit (see Figure 5 in [23]). Both case (2) and (3) generally agreed with the magnetic field findings for JO206 and built an highly ordered magnetic field in the tail that can be explained by the magnetic draping of the magnetised ICM field and the accretion of gas from that sheath. However, the homogeneous wind field naturally aligns with the tail and reaches; therefore, a non-physical high degree of polarization above $75 \%$ exists while field reversals in the turbulent wind field reduce magnetic tension. In the latter case, the tail magnetic field becomes aligned and stretched with the accretion of mixed warm gas to the cold phase resembling the observation parameters of JO206 most accurately.

\section{Holistic Picture}

The combined analysis of the different gas phases for a sub-sample of GASP galaxies and the unique polarization study of JO206, as well as the fact that radio tails are commonly found in ram-pressure stripped galaxies, puts forwards the idea that a highly ordered magnetic field may be common and play a crucial role in regulating star formation in the stripped tails. The following picture can be drawn (shown schematically in Figure 6). 


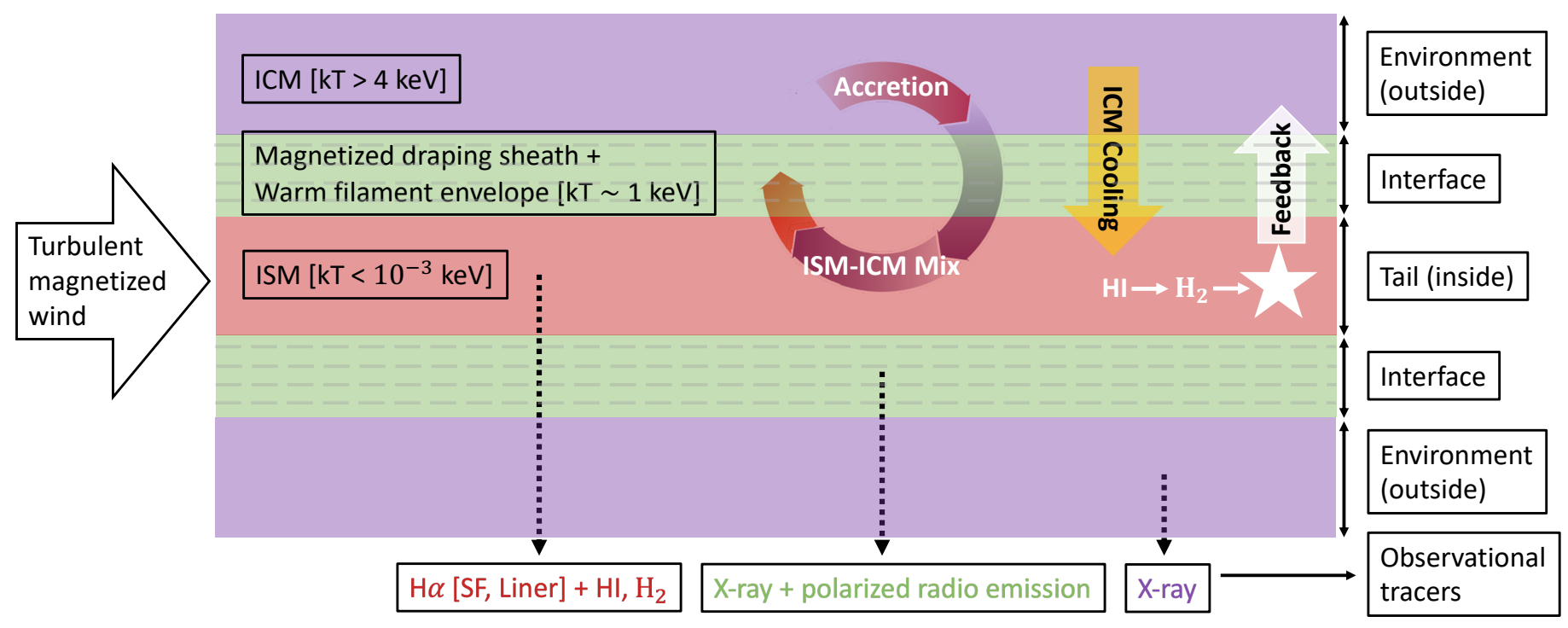

Figure 6. Simplistic view of a section of the stripped tail depicting the holistic picture in ram-pressure stripped galaxies. The ICM is shown as outer layers (purple), the magnetic field and accreted/condensed ICM below (green with grey dotted lines indicating the highly aligned draped magnetic field) and the ISM consisting of different gas phases in the inner layer (red). While ICM is accreted, joining the ISM via mixing (circle arrow), ICM cooling is taking place outside-in (yellow arrow). Effective $\mathrm{HI}$ to $\mathrm{H}_{2}$ conversion provides new stars that will provide feedback inside-out during stellar evolution and explosion processes (white arrow).

We found the observed highly ordered magnetic field far out into the tail of JO206 was in excellent agreement with magneto-hydrodynamic simulations of a gas cloud exposed to turbulent ICM magnetic field. The galaxy-sized cloud was found to accrete hot, draped magnetised plasma that further condenses onto the outer layers of the tail (formation of a warm envelope shown with green layer in Figure 6). Adiabatic compression and shearing by the velocity difference of the cold tail and the wind thereafter can explain the observed high degree of fractional polarization and ordered magnetic field in JO206. We propose a formation of a magnetic draping layer at the galaxies' leading edges and shearing along the length of the tail, which is indicated by the green layer in Figure 6 and the dotted line as aligned magnetic field. In such a scenario, the galaxy facing ram-pressure wind would be prevented from evaporating its cold ISM into the hot ICM (which is why the circle arrow stops inside the tail in Figure 6). Moreover, additional accretion of the cooled ICM from the outside-in (indicated by the yellow arrow in Figure 6) can provide new gas to form stars. The cooling timescales are still an open problem, however, given the physical context, non-equilibrium cooling processes are likely taking place (e.g., mixing). In order to investigate these non-linear processes, tailored numerical simulations are necessary. In addition to the JO206 observables, which already agree with such a scenario, we find more evidence in multi-phase gas complementing this view.

The magnetic field configuration and star formation rate should depend on the amount of gas that is stripped from the disc and accreted from the hot ICM. The highly ordered magnetic field is expected to enter a phase where it protects the stripped gas from the disk into the tail from evaporation into the ICM by preventing heat and momentum exchange between ISM and ICM. It is assumed that the accreted magnetised ICM cools while joining the cold-warm gaseous component of the ram-pressure stripped galaxy tails, possibly providing additional gas to form stars. Such a scenario cannot be probed by analysing only the magnetic field structure but different gas phases must also be investigated. More explicitly, hot ICM cooling along the tail during the accretion process should be observable in the X-ray band, explaining the extended X-ray emission found in different ram-pressure stripped tails (being part of the warm envelope shown in green in Figure 6). The thermal bremsstrahlung emission of the accreted gas could, in turn, be able to affect the ionized gas phases, as indicated by the excess in the ionization parameter 
([OI] $/ \mathrm{H} \alpha$, indicated by the inner red layer in Figure 6). Indeed, a tentative connection between thermal emission and the optical spectral properties in the stripped tails has been proposed in Campitiello et al. [36]. ${ }^{3}$

Further cooling would provide additional $\mathrm{HI}$ joining the stripped gas from the disk. The magnetic pressure and tail structure possibly have an impact on the increased transformation from $\mathrm{HI}$ to $\mathrm{H}_{2}$ and, therefore, the star-formation rates (shown by the white cartoon in Figure 6, where the white arrows indicate the effective transformation). In such a scenario, the magnetic field structure should be significantly ordered over larger time scales $\left(\sim 10^{8}\right.$ year) to prevent the tail gas from evaporation while the accreted ICM gas can effectively perform cooling. Within the star-forming regions, freshly ejected electrons join the magnetic field component mainly caused by the ICM (and also provide feedback inside-out, both indicated by the white arrow in Figure 6) but will not disrupt it; otherwise, gas is expected to evaporate into the ICM, the in situ star formation will stop eventually, and the galaxy evolves into a passive galaxy.

\section{Current Limitation and Future Perspective}

The recent results depict a close connection on a sub-kpc scale between the magnetic field and the ISM. Therefore, in order to investigate the physics of this link, high-resolution, sub-arcsecond radio observations are necessary. This is a current limitation that could potentially be solved in the near future by the sub-arcsecond imaging made possible by the international LOFAR stations [62]. A second crucial current limitation is the lack of candidates suitable for these studies, i.e., galaxies with both polarimetric and X-ray observations. In this regard, within GASP, we are working to expand this sample. We have already been awarded time to observe deep polarization for four more galaxiesJO204 and JW100 at the VLA, partly observed (2-4 GHz C-array, ID: 21A-248, PI: Ancla Müller); JO147, partly observed (1-3 GHz multiple array configurations, ID: C3357, PI: Ancla Müller); JO175 to be completed in Jan, 2022 (1-3 GHz multiple array configurations, ID: C3357, PI: Ancla Müller) —enabling us to probe the general existence of such a magnetic field configuration in star-forming, ram-pressure stripped tails. In combination with the deep Chandra observation (ID: 23610079, PI: Ignesti) of JO206 and its surrounding ICM, we will be able to unfold the possible impact of the magnetic field onto the entire multiphase gas.

Finally, the advent of deep radio surveys made possible by the new-generation facilities such as LOFAR and SKA in the future provide us the opportunity to investigate the properties of jellyfish magnetic fields on larger samples of galaxies and to evaluate the diversity of the cluster potential onto the connection of the magnetic field configuration and strength to the star-forming regions. Moreover, as outlined in $[27,28,46]$, low-frequency LOFAR observations provide us an unique insight into the low-energy electrons travelling along the stripped tails and the physical phenomena that drive their evolution. Future synergies between LOFAR, MeerkAT, VLA, and SKA will allow extending this kind of analysis to larger samples of galaxies, thus deepening our knowledge of these fascinating systems.

\section{Conclusions}

We provide a summary of the recent results collected within the GASP collaboration including the analysis of the cold neutral and molecular gas, diffuse ionized gas tracers, hot gas, radio continuum, and linear polarization. By combining the different observations, we propose a holistic view of the evolution of galaxies experiencing ram-pressure stripping. Here, we focus on the most massive galaxies and most elongated ram-pressure stripped tails forming stars in situ analysed within GASP. Both the diverse observations and recent simulations of a gas cloud facing a turbulent, hot, and magnetized ICM wind agree with a scenario in which the galaxy accretes hot plasma forming a magnetic draping layer at the leading edges and further along the tail preventing the stripped tail gas from evaporation while the ICM plasma becomes mixed with ISM and cools from the outside-in. On larger timescales, the tail might be able to add mass from the ICM, providing additional gas to 
possibly form new stars. We additionally argue that the formations of the magnetic draping sheath and the stars in the tail are self-regulating processes in which the outer layers will not survive due to a very efficient inside-out stellar feedback. We propose that the gas in the tails survives because of the combination of different effects: the galaxy mass, the galaxy infall velocity towards the cluster center, the pressure of the ICM surrounding the tails, and the formation of the magnetic layer protecting this gas from evaporation. The further study of the phenomena presented in this study will add an important piece of information to our knowledge of the outcomes of ram pressure stripping, as well as, more generally, to the scenario of draping, condensation, and cooling of hot gas surrounding cold clouds that is fundamental in many astrophysical phenomena.

Author Contributions: Conceptualization, A.M. (Ancla Müller), B.P., and A.I.; investigation, A.M. (Ancla Müller), B.P., and A.I.; writing-original draft preparation, A.M. (Ancla Müller) and A.I.; writing—review and editing, A.M. (Ancla Müller), A.I., B.P., A.M. (Alessia Moretti), M.R., and R.-J.D.; visualization, A.M. (Ancla Müller), A.I., A.M. (Alessia Moretti), and M.R.; supervision, A.M. (Ancla Müller), B.P., and R.-J.D.; project administration, A.M. (Ancla Müller). All authors have read and agreed to the published version of the manuscript.

Funding: This project has received funding from the European Research Council (ERC) under the European Union's Horizon 2020 research and innovation programme (grant agreement No. 833824). We acknowledge funding from agreement ASI-INAF n.2017-14-H.0. (PI A. Moretti). We acknowledge financial support provided by SKA South-Africa and the South African National Research Foundation.

Data Availability Statement: The data presented in this study are available upon request from the corresponding author.

Acknowledgments: Based on observations collected at the European Organization for Astronomical Research in the Southern Hemisphere under ESO programme 196.B-0578. The authors are grateful to the entire GASP team for their contribution and useful discussions.

Conflicts of Interest: The authors declare no conflict of interest.

\section{Notes}

https:/ / web.oapd.inaf.it/gasp/ accessed on 9 December 2021)

For more details and information on the collection of publications, see https:/ / web.oapd.inaf.it/gasp/ (accessed on 9 December 2021) A similar scenario was previously proposed also to explain the optical properties of the cold filaments observed at the center of relaxed, cool core clusters, e.g., [60,61].

\section{References}

1. Noeske, K.G.; Weiner, B.J.; Faber, S.M.; Papovich, C.; Koo, D.C.; Somerville, R.S.; Bundy, K.; Conselice, C.J.; Newman, J.A.; Schiminovich, D.; et al. Star Formation in AEGIS Field Galaxies since $z=1.1$ : The Dominance of Gradually Declining Star Formation, and the Main Sequence of Star-forming Galaxies. Astrophys. J. 2007, 660, L43-L46. [CrossRef]

2. Madau, P.; Dickinson, M. Cosmic Star-Formation History. Annu. Rev. Astron. Astrophys. 2014, 52, 415-486. [CrossRef]

3. Boselli, A.; Gavazzi, G. Environmental Effects on Late-Type Galaxies in Nearby Clusters. Publ. Astron. Soc. Pac. 2006, 118, 517-559. [CrossRef]

4. Cayatte, V.; van Gorkom, J.H.; Balkowski, C.; Kotanyi, C. VLA Observations of Neutral Hydrogen in Virgo Cluster Galaxies. I. The Atlas. Astron. J. 1990, 100, 604. [CrossRef]

5. Kenney, J.D.P.; van Gorkom, J.H.; Vollmer, B. VLA HiObservations of Gas Stripping in the Virgo Cluster Spiral NGC 4522. Astron. J. 2004, 127, 3361-3374. [CrossRef]

6. Chung, A.; van Gorkom, J.H.; Kenney, J.D.P.; Crowl, H.; Vollmer, B. VLA Imaging of Virgo Spirals in Atomic Gas (VIVA). I. The Atlas and the H I Properties. Astrophys. J. 2009, 138, 1741-1816. [CrossRef]

7. Jaffé, Y.L.; Smith, R.; Candlish, G.N.; Poggianti, B.M.; Sheen, Y.K.; Verheijen, M.A.W. BUDHIES II: A phase-space view of H I gas stripping and star formation quenching in cluster galaxies. Mon. Not. R. Astron. Soc. 2015, 448, 1715-1728. [CrossRef]

8. Scott, T.C.; Brinks, E.; Cortese, L.; Boselli, A.; Bravo-Alfaro, H. Abell 1367: A high fraction of late-type galaxies displaying H I morphological and kinematic perturbations. Mon. Not. R. Astron. Soc. 2018, 475, 4648-4669. [CrossRef]

9. Williams, B.A.; Rood, H.J. Neutral Hydrogen in Compact Groups of Galaxies. Astrophys. J. 1987, 63, 265. [CrossRef]

10. Verdes-Montenegro, L.; Yun, M.S.; Williams, B.A.; Huchtmeier, W.K.; Del Olmo, A.; Perea, J. Where is the neutral atomic gas in Hickson groups? Astron. Astrophys. 2001, 377, 812-826. [CrossRef] 
11. Serra, P.; Koribalski, B.; Duc, P.A.; Oosterloo, T.; McDermid, R.M.; Michel-Dansac, L.; Emsellem, E.; Cuillandre, J.C.; Alatalo, K.; Blitz, L.; et al. Discovery of a giant HI tail in the galaxy group HCG 44. Mon. Not. R. Astron. Soc. 2013, 428, 370-380. [CrossRef]

12. Bellhouse, C.; Jaffé, Y.L.; Hau, G.K.T.; McGee, S.L.; Poggianti, B.M.; Moretti, A.; Gullieuszik, M.; Bettoni, D.; Fasano, G.; D’Onofrio, M.; et al. GASP. II. A MUSE View of Extreme Ram-Pressure Stripping along the Line of Sight: Kinematics of the Jellyfish Galaxy JO201. Astrophys. J. 2017, 844, 49. [CrossRef]

13. Boselli, A.; Fossati, M.; Sun, M. Ram Pressure Stripping in High-Density Environments. arXiv 2021, arXiv:2109.13614.

14. Cortese, L.; Marcillac, D.; Richard, J.; Bravo-Alfaro, H.; Kneib, J.P.; Rieke, G.; Covone, G.; Egami, E.; Rigby, J.; Czoske, O.; et al. The strong transformation of spiral galaxies infalling into massive clusters at $\mathrm{z} \sim 0.2$. Mon. Not. R. Astron. Soc. 2007, 376, 157-172. [CrossRef]

15. Poggianti, B.M.; Gullieuszik, M.; Tonnesen, S.; Moretti, A.; Vulcani, B.; Radovich, M.; Jaffé, Y.; Fritz, J.; Bettoni, D.; Franchetto, A.; et al. GASP XIII. Star formation in gas outside galaxies. Mon. Not. R. Astron. Soc. 2019, 482, 4466-4502. [CrossRef]

16. Crutcher, R.M. Magnetic Fields in Molecular Clouds. Annu. Rev. Astron. Astrophys. 2012, 50, 29-63. [CrossRef]

17. Heesen, V.; Beck, R.; Krause, M.; Dettmar, R.J. Cosmic rays and the magnetic field in the nearby starburst galaxy NGC 253 III. Helical magnetic fields in the nuclear outflow. Astron. Astrophys. 2011, 535, A79. [CrossRef]

18. Beck, R.; Wielebinski, R. Magnetic Fields in Galaxies. In Planets, Stars and Stellar Systems. Volume 5: Galactic Structure and Stellar Populations; Springer: Berlin/Heidelberg, Germany, 2013; Volume 5, p. 641. [CrossRef]

19. Li, H.B.; Henning, T. The alignment of molecular cloud magnetic fields with the spiral arms in M33. Nature 2011, 479, 499-501. [CrossRef]

20. Gavazzi, G.; Contursi, A.; Carrasco, L.; Boselli, A.; Kennicutt, R.; Scodeggio, M.; Jaffe, W. The radio and optical structure of three peculiar galaxies in A 1367. Astron. Astrophys. 1995, 304, 325.

21. Vollmer, B.; Soida, M.; Chung, A.; Beck, R.; Urbanik, M.; Chyży, K.T.; Otmianowska-Mazur, K.; van Gorkom, J.H. The influence of the cluster environment on the large-scale radio continuum emission of 8 Virgo cluster spirals. Astron. Astrophys. 2010, 512 , A36. [CrossRef]

22. Chen, H.; Sun, M.; Yagi, M.; Bravo-Alfaro, H.; Brinks, E.; Kenney, J.; Combes, F.; Sivanandam, S.; Jachym, P.; Fossati, M.; et al. The ram pressure stripped radio tails of galaxies in the Coma cluster. Mon. Not. R. Astron. Soc. 2020, 496, 4654-4673. [CrossRef]

23. Müller, A.; Poggianti, B.M.; Pfrommer, C.; Adebahr, B.; Serra, P.; Ignesti, A.; Sparre, M.; Gitti, M.; Dettmar, R.J.; Vulcani, B.; et al. Highly ordered magnetic fields in the tail of the jellyfish galaxy JO206. Nat. Astron. 2021, 5, 159-168. [CrossRef]

24. Ignesti, A.; Vulcani, B.; Poggianti, B.M.; Paladino, R.; Shimwell, T.; Healy, J.; Gitti, M.; Bacchini, C.; Moretti, A.; Radovich, M.; et al. GASP XXXVIII: The LOFAR-MeerKAT-VLA view on the non-thermal side of a jellyfish galaxy. arXiv 2021, arXiv:2110.12719.

25. Poggianti, B.M.; Ignesti, A.; Gitti, M.; Wolter, A.; Brighenti, F.; Biviano, A.; George, K.; Vulcani, B.; Gullieuszik, M.; Moretti, A.; et al. GASP XXIII: A Jellyfish Galaxy as an Astrophysical Laboratory of the Baryonic Cycle. Astrophys. J. 2019, 887, 155. [CrossRef]

26. Shimwell, T.W.; Röttgering, H.J.A.; Best, P.N.; Williams, W.L.; Dijkema, T.J.; de Gasperin, F.; Hardcastle, M.J.; Heald, G.H.; Hoang, D.N.; Horneffer, A.; et al. The LOFAR Two-metre Sky Survey. I. Survey description and preliminary data release. Astron. Astrophys. 2017, 598, A104. [CrossRef]

27. Roberts, I.D.; van Weeren, R.J.; McGee, S.L.; Botteon, A.; Drabent, A.; Ignesti, A.; Rottgering, H.J.A.; Shimwell, T.W.; Tasse, C. LoTSS jellyfish galaxies. Astron. Astrophys. 2021, 650, A111. [CrossRef]

28. Roberts, I.D.; van Weeren, R.J.; McGee, S.L.; Botteon, A.; Ignesti, A.; Rottgering, H.J.A. LoTSS jellyfish galaxies: II. Ram pressure stripping in groups versus clusters. arXiv 2021, arXiv:2106.06315.

29. Poggianti, B.M.; Moretti, A.; Gullieuszik, M.; Fritz, J.; Jaffé, Y.; Bettoni, D.; Fasano, G.; Bellhouse, C.; Hau, G.; Vulcani, B.; Biviano, A.; et al. GASP. I. Gas Stripping Phenomena in Galaxies with MUSE. Astrophys. J. 2017, 844, 48. [CrossRef]

30. Poggianti, B.M.; Fasano, G.; Omizzolo, A.; Gullieuszik, M.; Bettoni, D.; Moretti, A.; Paccagnella, A.; Jaffé, Y.L.; Vulcani, B.; Fritz, J.; et al. Jellyfish Galaxy Candidates at Low Redshift. Astron. J. 2016, 151, 78. [CrossRef]

31. Ramatsoku, M.; Serra, P.; Poggianti, B.M.; Moretti, A.; Gullieuszik, M.; Bettoni, D.; Deb, T.; Fritz, J.; van Gorkom, J.H.; Jaffe, Y.L.; et al. GASP-XVII. H I imaging of the jellyfish galaxy JO206: Gas stripping and enhanced star formation. Mon. Not. R. Astron. Soc. 2019, 487, 4580-4591. [CrossRef]

32. Ramatsoku, M.; Serra, P.; Poggianti, B.M.; Moretti, A.; Gullieuszik, M.; Bettoni, D.; Deb, T.; Franchetto, A.; van Gorkom, J.H.; Jaffé, Y.; et al. GASP. XXVI. HI gas in jellyfish galaxies: The case of JO201 and JO206. Astron. Astrophys. 2020, 640, A22. [CrossRef]

33. Deb, T.; Verheijen, M.A.W.; Gullieuszik, M.; Poggianti, B.M.; van Gorkom, J.H.; Ramatsoku, M.; Serra, P.; Moretti, A.; Vulcani, B.; Bettoni, D.; et al. GASP XXV: Neutral hydrogen gas in the striking jellyfish galaxy JO204. Mon. Not. R. Astron. Soc. 2020, 494, 5029-5043. [CrossRef]

34. Moretti, A.; Paladino, R.; Poggianti, B.M.; D’Onofrio, M.; Bettoni, D.; Gullieuszik, M.; Jaffé, Y.L.; Vulcani, B.; Fasano, G.; Fritz, J.; et al. GASP-X. APEX observations of molecular gas in the discs and in the tails of ram-pressure stripped galaxies. Mon. Not. R. Astron. Soc. 2018, 480, 2508-2520. [CrossRef]

35. Moretti, A.; Paladino, R.; Poggianti, B.M.; Serra, P.; Ramatsoku, M.; Franchetto, A.; Deb, T.; Gullieuszik, M.; Tomičić, N.; Mingozzi, M.; et al. The High Molecular Gas Content, and the Efficient Conversion of Neutral into Molecular Gas, in Jellyfish Galaxies. Astrophys. J. 2020, 897, L30. [CrossRef]

36. Campitiello, M.G.; Ignesti, A.; Gitti, M.; Brighenti, F.; Radovich, M.; Wolter, A.; Tomičić, N.; Bellhouse, C.; Poggianti, B.M.; Moretti, A.; et al. GASP XXXIV: Unfolding the Thermal Side of Ram Pressure Stripping in the Jellyfish Galaxy JO201. Astrophys. J. 2021, 911, 144. [CrossRef] 
37. Tomicic, N.; Vulcani, B.; Poggianti, B.M.; Werle, A.; Muller, A.; Mingozzi, M.; Gullieuszik, M.; Wolter, A.; Radovich, M.; Moretti, A.; et al. GASP XXXV: Characteristics of the diffuse ionised gas in gas-stripped galaxies. arXiv 2021, arXiv:2108.12433.

38. Sun, M.; Ge, C.; Luo, R.; Yagi, M.; Jáchym, P.; Boselli, A.; Fossati, M.; Nulsen, P.E.J.; Yoshida, M.; Gavazzi, G. Tales of tails: $\mathrm{H} \alpha$-X-ray correlation. arXiv 2021, arXiv:2103.09205.

39. Jaffé, Y.L.; Poggianti, B.M.; Moretti, A.; Gullieuszik, M.; Smith, R.; Vulcani, B.; Fasano, G.; Fritz, J.; Tonnesen, S.; Bettoni, D.; et al. GASP. IX. Jellyfish galaxies in phase-space: An orbital study of intense ram-pressure stripping in clusters. Mon. Not. R. Astron. Soc. 2018, 476, 4753-4764. [CrossRef]

40. Ignesti, A.; Gitti, M.; Brunetti, G.; O'Sullivan, E.; Sarazin, C.; Wong, K. The mystery of the "Kite" radio source in Abell 2626: Insights from new Chandra observations. Astron. Astrophys. 2018, 610, A89. [CrossRef]

41. Strickland, D.K.; Heckman, T.M.; Colbert, E.J.M.; Hoopes, C.G.; Weaver, K.A. A High Spatial Resolution X-ray and H $\alpha$ Study of Hot Gas in the Halos of Star-forming Disk Galaxies. I. Spatial and Spectral Properties of the Diffuse X-ray Emission. Astrophys. J. 2004, 151, 193-236. [CrossRef]

42. Moretti, A.; Paladino, R.; Poggianti, B.M.; Serra, P.; Roediger, E.; Gullieuszik, M.; Tomičić, N.; Radovich, M.; Vulcani, B.; Jaffé, Y.L.; et al. GASP. XXII. The Molecular Gas Content of the JW100 Jellyfish Galaxy at z 0.05: Does Ram Pressure Promote Molecular Gas Formation? Astrophys. J. 2020, 889, 9. [CrossRef]

43. Müller, A.; Pfrommer, C.; Ignesti, A.; Moretti, A.; Lourenço, A.; Paladino, R.; Jaffé, Y.; Gitti, M.; Venturi, T.; Gullieuszik, M.; et al. Two striking head-tail galaxies in the galaxy cluster IIZW108: Insights into transition to turbulence, magnetic fields, and particle re-acceleration. Mon. Not. R. Astron. Soc. 2021, 508, 5326-5344. [CrossRef]

44. Gitti, M. The puzzling radio source in the cool core cluster A2626. Mon. Not. R. Astron. Soc. 2013, 436, L84-L88. [CrossRef]

45. Ignesti, A.; Gitti, M.; Brunetti, G.; Feretti, L.; Giovannini, G. New JVLA observations at $3 \mathrm{GHz}$ and $5.5 \mathrm{GHz}$ of the "Kite” radio source in Abell 2626. Astron. Astrophys. 2017, 604, A21. [CrossRef]

46. Ignesti, A.; Shimwell, T.; Brunetti, G.; Gitti, M.; Intema, H.; van Weeren, R.J.; Hardcastle, M.J.; Clarke, A.O.; Botteon, A.; Di Gennaro, G.; et al. The great Kite in the sky: A LOFAR observation of the radio source in Abell 2626. Astron. Astrophys. 2020, 643, A172. [CrossRef]

47. Tonnesen, S.; Stone, J. The Ties that Bind? Galactic Magnetic Fields and Ram Pressure Stripping. Astrophys. J. 2014, 795, 148. [CrossRef]

48. Ramos-Martínez, M.; Gómez, G.C.; Pérez-Villegas, Á. MHD simulations of ram pressure stripping of a disc galaxy. Mon. Not. R. Astron. Soc. 2018, 476, 3781-3792. [CrossRef]

49. Vollmer, B.; Soida, M.; Beck, R.; Urbanik, M.; Chyży, K.T.; Otmianowska-Mazur, K.; Kenney, J.D.P.; van Gorkom, J.H. The characteristic polarized radio continuum distribution of cluster spiral galaxies. Astron. Astrophys. 2007, 464, L37-L40. [CrossRef]

50. Dursi, L.J.; Pfrommer, C. Draping of Cluster Magnetic Fields over Bullets and Bubbles-Morphology and Dynamic Effects. Astrophys. J. 2008, 677, 993-1018. [CrossRef]

51. Pfrommer, C.; Dursi, L.J. Detecting the orientation of magnetic fields in galaxy clusters. Nat. Phys. 2010, 6, 520-526. [CrossRef]

52. Ruszkowski, M.; Brüggen, M.; Lee, D.; Shin, M.S. Impact of Magnetic Fields on Ram Pressure Stripping in Disk Galaxies. Astrophys. J. 2014, 784, 75. [CrossRef]

53. Berlok, T.; Pfrommer, C. The impact of magnetic fields on cold streams feeding galaxies. Mon. Not. R. Astron. Soc. 2019, 489, 3368-3384. [CrossRef]

54. Springel, V. Moving-mesh hydrodynamics with the AREPO code. Proc. Int. Astron. Union 2010, 6, 203-206. [CrossRef]

55. Pakmor, R.; Springel, V. Simulations of magnetic fields in isolated disc galaxies. Mon. Not. R. Astron. Soc. 2013, 432, 176-193. [CrossRef]

56. Pakmor, R.; Springel, V.; Bauer, A.; Mocz, P.; Munoz, D.J.; Ohlmann, S.T.; Schaal, K.; Zhu, C. Improving the convergence properties of the moving-mesh code AREPO. Mon. Not. R. Astron. Soc. 2015, 455, 1134-1143. [CrossRef]

57. Sparre, M.; Pfrommer, C.; Ehlert, K. Interaction of a cold cloud with a hot wind: The regimes of cloud growth and destruction and the impact of magnetic fields. arXiv 2020, arXiv:2008.09118.

58. Gronke, M.; Oh, S.P. The growth and entrainment of cold gas in a hot wind. Mon. Not. R. Astron. Soc. 2018, 480, L111-L115. [CrossRef]

59. Li, Z.; Hopkins, P.F.; Squire, J.; Hummels, C. On the survival of cool clouds in the circumgalactic medium. Mon. Not. R. Astron. Soc. 2020, 492, 1841-1854. [CrossRef]

60. McDonald, M.; Veilleux, S.; Rupke, D.S.N. Optical Spectroscopy of Halpha Filaments in Cool Core Clusters: Kinematics, Reddening, and Sources Of Ionization. Astrophys. J. 2012, 746, 153. [CrossRef]

61. Olivares, V.; Salome, P.; Combes, F.; Hamer, S.; Guillard, P.; Lehnert, M.D.; Polles, F.L.; Beckmann, R.S.; Dubois, Y.; Donahue, M.; et al. Ubiquitous cold and massive filaments in cool core clusters. Astron. Astrophys. 2019, 631, A22. [CrossRef]

62. Morabito, L.K.; Jackson, N.J.; Mooney, S.; Sweijen, F.; Badole, S.; Kukreti, P.; Venkattu, D.; Groeneveld, C.; Kappes, A.; Bonnassieux, E.; et al. Sub-arcsecond imaging with the International LOFAR Telescope I. Foundational calibration strategy and pipeline. arXiv 2021, arXiv:2108.07283. 